\title{
Intuitionistic Fuzzy Normalized Weighted Bonferroni Mean and Its Application in Multicriteria Decision Making
}

\author{
Wei Zhou' ${ }^{1,2}$ and Jian-min $\mathrm{He}^{2}$ \\ ${ }^{1}$ International Business School, Yunnan University of Finance and Economics, Kunming 650221, China \\ ${ }^{2}$ School of Economics and Management, Southeast University, Nanjing 211189, China \\ Correspondence should be addressed to Wei Zhou, zw453@163.com
}

Received 15 April 2012; Revised 28 June 2012; Accepted 10 July 2012

Academic Editor: Tak-Wah Lam

Copyright (c) 2012 W. Zhou and J.-m. He. This is an open access article distributed under the Creative Commons Attribution License, which permits unrestricted use, distribution, and reproduction in any medium, provided the original work is properly cited.

The Bonferroni mean (BM) was introduced by Bonferroni six decades ago but has been a hot research topic recently since its usefulness of the aggregation techniques. The desirable characteristic of the BM is its capability to capture the interrelationship between input arguments. However, the classical BM and GBM ignore the weight vector of aggregated arguments, the general weighted BM (WBM) has not the reducibility, and the revised generalized weighted BM (GWBM) cannot reflect the interrelationship between the individual criterion and other criteria. To deal with these issues, in this paper, we propose the normalized weighted Bonferroni mean (NWBM) and the generalized normalized weighted Bonferroni mean (GNWBM) and study their desirable properties, such as reducibility, idempotency, monotonicity, and boundedness. Furthermore, we investigate the NWBM and GNWBM operators under the intuitionistic fuzzy environment which is more common phenomenon in modern life and develop two new intuitionistic fuzzy aggregation operators based on the NWBM and GNWBM, that is, the intuitionistic fuzzy normalized weighted Bonferroni mean (IFNWBM) and the generalized intuitionistic fuzzy normalized weighted Bonferroni mean (GIFNWBM). Finally, based on the GIFNWBM, we propose an approach to multicriteria decision making under the intuitionistic fuzzy environment, and a practical example is provided to illustrate our results.

\section{Introduction}

Multicriteria decision making is the pervasive phenomenon in modern life, which is to select the best or optimal alternative from several alternatives or to get their ranking by aggregating the performances of each alternative under some criteria, in which the aggregation operators play an important role. As many different types of criteria relationships exist in the real world 
there is a need for many types of formal aggregation operations to enable the modeling of these numerous types of relationships. In response to this need a formal mathematical discipline called aggregation theory is emerging [1-4]. In this paper, we contribute to this theory by looking at the Bonferroni mean (BM), proposing the normalized weighted BM (NWBM) and the generalized normalized weighted BM (GNWBM) and developing the generalized intuitionistic fuzzy normalized weighted BM (GIFNWBM) and its application in multicriteria decision making.

Bonferroni [5] originally introduced a mean-type aggregation operator called the Bonferroni mean, which can provide for the aggregation lying between the max and min operators and logical "oring" and "anding" operators. A prominent characteristic of $\mathrm{BM}$ is that it cannot only consider the importance of each criterion but also reflect the interrelationship of the individual criterion. Recently, Yager [6] further studied the BM and provided an interpretation of $\mathrm{BM}$ as involving a product of each argument with the average of the other arguments, and where the BM was shown to be suitable for modeling various concepts, such as hard and soft partial conjunction and disjunction [7] and boundedness similar to $k$-intolerance $[8,9]$. Furthermore, Yager [6] extends the BM replacing the simple average by other mean-type operators, such as the Choquet integral [10] and the ordered weighted averaging operator [11], as well as associates differing importance with the arguments. Mordelová and Rückschlossová [12] also investigated the generalizations of BM referred to as ABC-aggregation functions. Beliakov et al. [1] further extended the BM by considering the correlations of any three aggregated arguments instead of any two and proposed the generalized Bonferroni mean (GBM). Nevertheless, the arguments suitable to be aggregated by the BM and GBM can only take the forms of crisp numbers rather than any other types of arguments, which restrict the potential applications of the BM to more extensive areas. In the real world, due to the increasing complexity of the socioeconomic environment and the lack of knowledge and data, crisp data are sometimes unavailable. Thus, the input arguments may be more suitable with representation of fuzzy formats, such as fuzzy number [13], interval-valued fuzzy number [14], intuitionistic fuzzy value [15], interval-valued intuitionistic fuzzy value [16], and hesitant fuzzy element $[17,18]$. Therefore, $\mathrm{Xu}$ and Yager [19] applied the BM to intuitionistic fuzzy environment and introduced the intuitionistic fuzzy Bonferroni mean (IFBM) and the intuitionistic fuzzy weighted Bonferroni mean (IFWBM), $\mathrm{Xu}$ and Chen [20] further applied the BM to interval-valued intuitionistic fuzzy environment and introduced the interval-valued intuitionistic fuzzy Bonferroni mean (IIFBM) and the interval-valued intuitionistic fuzzy weighted Bonferroni mean (IIFWBM).

It is noted that the BM, GBM, IFBM, and IIFBM ignore the weight vector of the aggregated arguments, although the IFWBM and the IIFWBM consider this issue, we cannot, respectively, obtain IFBM and IIFBM when all the weights of the aggregated arguments are the same, that is, these two operators have not reducibility, which seems to be counterintuitive. To deal with this issue, Xia et al. [21] proposed the revised BM and revised generalized weighted Bonferroni mean (GWBM), which take into the weight vector and reducibility and extended them to intuitionistic fuzzy environment. However, a question arises, that is, the revised BM and the GWBM just reflect the correlationship between the individual criterion and all criteria, which is not an interrelationship between the individual criterion and other criteria represented in the BM. Therefore, to further develop BM, we propose the normalized weighted BM (NWBM) and the generalized normalized weighted BM (GNWBM). The main advantage of the NWBM and the GNWBM is that they can not only consider weight vector and interrelationship of the individual criterion which is similar to the IFWBM and the IIFWBM but also have the reducibility like the GWBM. Based on the NWBM 
and GNWBM operators, we develop the intuitionistic fuzzy normalized weighted Bonferroni mean (IFNWBM) and the generalized intuitionistic fuzzy normalized weighted Bonferroni mean (GIFNWBM), on the basis of which an approach to multicriteria decision making is also proposed.

The remainder of this paper is organized as follows. We briefly review some basic concepts and operations of the IFV and BM, in Section 2. Section 3 proposes the NWBM and GNWBM operators and studies their desirable properties. Section 4, the IFNWBM operator is proposed, and then its corresponding generalized form is also given. A practical example is provided in Section 5 to demonstrate the application of the generalized intuitionistic fuzzy normalized weighted Bonferroni mean. The paper ends in Section 6 with concluding remarks.

\section{Basic Concepts and Operations}

In this section, we introduce some basic notions and operations related to the intuitionistic fuzzy value and the Bonferroni mean.

\subsection{Intuitionistic Fuzzy Values}

Definition 2.1 (see [15]). Let $X=\left(x_{1}, x_{2}, \ldots, x_{n}\right)$ be fixed. An intuitionistic fuzzy set (IFS) $A$ in $X$ can be defined as

$$
A=\left\{\left(x_{i}, \mu\left(x_{i}\right), v\left(x_{i}\right)\right) \mid x_{i} \in X\right\},
$$

where $\mu\left(x_{i}\right) \in[0,1]$ and $v\left(x_{i}\right) \in[0,1]$ satisfy $0 \leq \mu\left(x_{i}\right)+v\left(x_{i}\right) \leq 1$ for all $x_{i} \in X$ and $\mu\left(x_{i}\right)$ and $v\left(x_{i}\right)$ are, respectively, called the degree of membership and the degree of nonmembership of the element $x_{i} \in X$ to $A$.

Furthermore, $\pi\left(x_{i}\right)=1-\mu\left(x_{i}\right)+v\left(x_{i}\right)$ is called the hesitation degree of $x_{i}$ to $A$, which represents the indeterminacy degree. For computational convenience, $\mathrm{Xu}$ [22] named the pair $\left(\mu_{\alpha}, v_{\alpha}\right)$ an intuitionistic fuzzy value (IFV) denoted as $\alpha$ with the conditions $0 \leq \mu_{\alpha} \leq 1,0 \leq$ $v_{\alpha} \leq 1$ and $0 \leq \mu\left(x_{i}\right)+v\left(x_{i}\right) \leq 1$. The set of IFVs is denoted as $\Omega$. To compare and calculate the IFVs, Chen and Tan [23] introduced the score function $s(\alpha)=\mu_{\alpha}-v_{\alpha}$ to get the score value of $\alpha$, and Hong and Choi [24] defined the accuracy function $h(\alpha)=\mu_{\alpha}+v_{\alpha}$ to evaluate the accuracy degree of $\alpha$. Based on the score function and the accuracy function, $\mathrm{Xu}$ and Yager [25] gave a total order relation between two IFVs $\alpha$ and $\beta$, as follows:

$$
\begin{aligned}
& \text { if } s(\alpha)<s(\beta), \text { then } \alpha<\beta ; \\
& \text { if } s(\alpha)=s(\beta), \text { then }
\end{aligned}
$$

(i) if $h(\alpha)=h(\beta)$, then $\alpha=\beta$; (ii) if $h(\alpha)<h(\beta)$, then $\alpha<\beta$.

Definition 2.2 (see [22, 25]). Let $\alpha=\left(\mu_{\alpha}, v_{\alpha}\right), \alpha_{1}=\left(\mu_{\alpha_{1}}, v_{\alpha_{1}}\right)$, and $\alpha_{2}=\left(\mu_{\alpha_{2}}, v_{\alpha_{2}}\right)$ be three IFVs, then following operational laws are valid:

(1) $\alpha_{1} \oplus \alpha_{2}=\left(\mu_{\alpha_{1}}+\mu_{\alpha_{2}}-\mu_{\alpha_{1}} \cdot \mu_{\alpha_{2}}, v_{\alpha_{1}} \cdot v_{\alpha_{2}}\right)$;

(2) $\alpha_{1} \otimes \alpha_{2}=\left(\mu_{\alpha_{1}} \cdot \mu_{\alpha_{2}}, v_{\alpha_{1}}+v_{\alpha_{2}}-v_{\alpha_{1}} \cdot v_{\alpha_{2}}\right)$;

(3) $\lambda \alpha=\left(1-\left(1-\mu_{\alpha}\right)^{\lambda}, v_{\alpha}^{\lambda}\right), \lambda>0$;

(4) $\alpha^{\lambda}=\left(\mu_{\alpha}^{\lambda}, 1-\left(1-v_{\alpha}\right)^{\lambda}\right), \lambda>0$. 


\subsection{Bonferroni Means}

The Bonferroni mean was originally introduced by Bonferroni [5] and intensively investigated by Yager [6], which was defined as follows.

Definition 2.3 (see [5]). Let $p, q \geq 0$ and $a_{i}(i=1,2, \ldots, n)$ be a collection of nonnegative numbers. If

$$
\operatorname{BM}^{p, q}\left(a_{1}, a_{2}, \ldots, a_{n}\right)=\left(\frac{1}{n(n-1)} \sum_{\substack{i, j=1 \\ i \neq j}}^{n} a_{i}^{p} a_{j}^{q}\right)^{1 /(p+q)}
$$

then $\mathrm{BM}^{p, q}$ is called the Bonferroni mean (BM).

One interpretation of the Bonferroni Mean is as a kind of combined "anding" and "averaging" operator [6]. Then, here we see that $a_{i}^{p} a_{j}^{q}$ indicates the degree to which both criteria $A_{i}$ and $A_{j}$ are satisfied under the given conditions and the special case when $p=q=$ 1 . There exists another interesting way to view this aggregation operator and described as follows.

$$
\operatorname{BM}^{p, q}\left(a_{1}, a_{2}, \ldots, a_{n}\right)=\left(\frac{1}{n} \sum_{i=1}^{n} a_{i}^{p}\left(\frac{1}{n-1} \sum_{\substack{j=1 \\ j \neq i}}^{n} a_{j}^{q}\right)\right)^{1 /(p+q)} .
$$

We see that the term $(1 /(n-1)) \sum_{j=1, j \neq i}^{n} a_{j}^{q}$ is the power average satisfaction of all criteria except $A_{i}$. We will denote this as $u_{i}^{q}$. Thus

$$
\operatorname{BM}^{p, q}\left(a_{1}, a_{2}, \ldots, a_{n}\right)=\left(\frac{1}{n} \sum_{i=1}^{n} a_{i}^{p} u_{i}^{q}\right)^{1 /(p+q)} .
$$

Here then $u_{i}^{q}$ is the power average satisfaction to all criteria except $A_{i}$ and $a_{i}^{p} u_{i}^{q}$ represent the interrelationship between $A_{i}$ and other criteria $A_{j}$, which is also the prominent characteristic of the BM. Based on the BM, Beliakov et al. [1] further extended and generalized the $\mathrm{BM}$ to the generalized Bonferroni mean (GBM) by considering the correlations of any three aggregated arguments instead of any two.

Definition 2.4 (see [1]). Let $p, q, r \geq 0$ and $a_{i}(i=1,2, \ldots, n)$ be a collection of nonnegative numbers. If

$$
\operatorname{GBM}^{p, q, r}\left(a_{1}, a_{2}, \ldots, a_{n}\right)=\left(\frac{1}{n(n-1)(n-2)} \sum_{\substack{i, j, k=1 \\ i \neq j \neq k}}^{n} a_{i}^{p} a_{j}^{q} a_{k}^{r}\right)^{1 /(p+q+r)}
$$

then $\mathrm{GBM}^{p, q, r}$ is called the generalized Bonferroni mean (GBM). 
It is obvious that the GBM reduces to the BM if $r=0$, and the GBM can represent the interrelationship of any three criteria. Here, we see that the term $1 /(n-1) \sum_{j=1, i \neq j}^{n}(1 /(n-$ 2) $\left.\sum_{k=1, k \neq i \neq j}^{n} a_{j}^{q} a_{k}^{r}\right)$ is the power average satisfaction of all criteria correlationship except $A_{i}$, denote as $v_{i}^{q, r}$. Thus

$$
\operatorname{GBM}^{p, q, r}\left(a_{1}, a_{2}, \ldots, a_{n}\right)=\left(\frac{1}{n} \sum_{i=1}^{n} a_{i}^{p} v_{i}^{q, r}\right)^{1 /(p+q+r)} .
$$

The above BM and GBM can only deal with the situation that the arguments are represented by real number but are invalid if the aggregation information is given in other forms, such as the IFV, which is a widely used technique to deal with uncertainty and vagueness. To deal with this issue, $\mathrm{Xu}$ and Yager [19] extended the BM to intuitionistic fuzzy environment and gave the following definition.

Definition 2.5 (see [19]). Let $p, q, r \geq 0$, and $\alpha_{i}(i=1,2, \ldots, n)$ be a collection of intuitionistic fuzzy values. The intuitionistic fuzzy Bonferroni mean (IFBM) and the intuitionistic fuzzy weighted Bonferroni mean (IFWBM) are, respectively, defined as

$$
\begin{gathered}
\operatorname{IFBM}^{p, q}\left(\alpha_{1}, \alpha_{2}, \ldots, \alpha_{n}\right)=\left(\frac{1}{n(n-1)} \bigoplus_{i, j=1, i \neq j}^{n}\left(\alpha_{i}^{p} \otimes \alpha_{j}^{q}\right)\right)^{1 /(p+q)}, \\
\operatorname{IFWBM}^{p, q}\left(\alpha_{1}, \alpha_{2}, \ldots, \alpha_{n}\right)=\left(\frac{1}{n(n-1)} \bigoplus_{i, j=1, i \neq j}^{n}\left(\left(w_{i} \alpha_{i}^{p}\right) \otimes\left(w_{j} \alpha_{j}^{q}\right)\right)\right)^{1 /(p+q)} .
\end{gathered}
$$

However, it is noted that the BM, GBM, and IFBM ignore the weight vector of the aggregated arguments, although the IFWBM considers this issue, we cannot obtain the IFBM when all the weights of the aggregated arguments are the same, that is, these IFWBM operator has not the reducibility, which seems to be counterintuitive. Therefore, to deal with these issues, Xia et al. [21] proposed the generalized weighted Bonferroni mean (GWBM) based on the GBM and described as follows.

Definition 2.6 (see [21]). Let $p, q \geq 0$, and $a_{i}(i=1,2, \ldots, n)$ be a collection of nonnegative numbers with the weight vector $w=\left(w_{1}, w_{2}, \ldots, w_{n}\right)$ such that $w_{i} \geq 0$ and $\sum_{i=1}^{n} w_{i}=1$. If

$$
\operatorname{GWBM}^{p, q, r}\left(a_{1}, a_{2}, \ldots, a_{n}\right)=\left(\sum_{i, j, k=1}^{n} w_{i} w_{j} w_{k} a_{i}^{p} a_{j}^{q} a_{k}^{r}\right)^{1 /(p+q+r)}
$$

then $\mathrm{GWBM}^{p, q, r}$ is called the generalized weighted Bonferroni mean (GWBM). 
If $w=(1 / n, 1 / n, \ldots, 1 / n)$, then the GWBM reduces to the revised GBM, that is,

$$
\operatorname{GBM}^{p, q, r}\left(a_{1}, a_{2}, \ldots, a_{n}\right)=\left(\frac{1}{n^{3}} \sum_{i, j, k=1}^{n} a_{i}^{p} a_{j}^{q} a_{k}^{r}\right)^{1 /(p+q+r)}
$$

which reflects the reducibility.

Similarly, we can transform the GWBM into the following form:

$$
\operatorname{GBM}^{p, q, r}\left(a_{1}, a_{2}, \ldots, a_{n}\right)=\left(\frac{1}{n} \sum_{i=1}^{n} a_{i}^{p}\left(\frac{1}{n} \sum_{j=1}^{n} a_{j}^{q}\right)\left(\frac{1}{n} \sum_{k=1}^{n} a_{k}^{r}\right)\right)^{1 /(p+q+r)} .
$$

However, a question arises, that is, the GWBM just considers the whole correlationship between the criterion $a_{i}$ and all criteria $\sum_{j=1}^{n} a_{j}^{q} \cdot \sum_{k=1}^{n} a_{k}^{r}$ and cannot reflect the interrelationship between the individual criterion $a_{i}$ and other criteria $v_{i}^{q, r}$ which is the main advantage of the BM. To further overcome this drawback, we propose the following NWBM and GNWBM operators.

\section{Normalized Weighted BM and Generalized Normalized Weighted BM}

The classical BM and GBM ignore the weight vector of aggregated arguments, the general weighted BMs (WBM) have not reducibility, and the revised generalized BM (GWBM) cannot reflect the interrelationship between the individual criterion and other criteria. To deal with these issues, in the following subsections, we propose the normalized weighted versions of $\mathrm{BM}$ and GBM, that is, the normalized weighted BM (NWBM) and the generalized normalized weighted BM (GNWBM).

\subsection{NWBM}

Definition 3.1. Let $p, q \geq 0$ and $a_{i}(i=1,2, \ldots, n)$ be a collection of nonnegative numbers with the weight vector $w=\left(w_{1}, w_{2}, \ldots, w_{n}\right)$ such that $w_{i} \geq 0$, and $\sum_{i=1}^{n} w_{i}=1$. If

$$
\operatorname{NWBM}^{p, q}\left(a_{1}, a_{2}, \ldots, a_{n}\right)=\left(\sum_{\substack{i, j=1 \\ i \neq j}}^{n} \frac{w_{i} w_{j}}{1-w_{i}} a_{i}^{p} a_{j}^{q}\right)^{1 /(p+q)}
$$

then $\mathrm{NWBM}^{p, q}$ is called the normalized weighted Bonferroni mean (NWBM). 
Then, we can transform the NWBM into the interrelationship NWBM form as follows:

$$
\operatorname{NWBM}^{p, q}\left(a_{1}, a_{2}, \ldots, a_{n}\right)=\left(\sum_{i=1}^{n} w_{i} a_{i}^{p} \sum_{\substack{j=1 \\ j \neq i}}^{n} \frac{w_{j}}{1-w_{i}} a_{j}^{q}\right)^{1 /(p+q)}
$$

We see that the term $\sum_{j=1, j \neq i}^{n}\left(w_{j} /\left(1-w_{i}\right)\right) a_{j}^{q}$ is the weighted power average satisfaction of all criteria except $A_{i}$ and $\sum_{j=1, j \neq i}^{n} w_{j} /\left(1-w_{i}\right)=1$. We denote the term as $u_{i}^{q}$. Thus

$$
\operatorname{NWBM}^{p, q}\left(a_{1}, a_{2}, \ldots, a_{n}\right)=\left(\sum_{i=1}^{n} w_{i} a_{i}^{p} u_{i}^{q}\right)^{1 /(p+q)}
$$

Here then $u_{i}^{q}$ is the weighted power average satisfaction to all criteria except $A_{i}$, and $\mathrm{NWBM}^{p, q}$ represents the interrelationship between the individual criterion $a_{i}$ and other criteria $a_{j}(j \neq i)$ which is similar to the BM.

Moreover, the NWBM has the following properties.

Property 1 (Reducibility). Let $p, q \geq 0$ and $a_{i}(i=1,2, \ldots, n)$ be a collection of nonnegative numbers with the weight vector $w=(1 / n, 1 / n, \ldots 1 / n)$, then

$$
\operatorname{NWBM}^{p, q}\left(a_{1}, a_{2}, \ldots, a_{n}\right)=\operatorname{BM}^{p, q}\left(a_{1}, a_{2}, \ldots, a_{n}\right)
$$

Proof. Since $w=(1 / n, 1 / n, \ldots 1 / n)$, then by Definition 3.1, we have

$$
\begin{aligned}
\operatorname{NWBM}^{p, q}\left(a_{1}, a_{2}, \ldots, a_{n}\right) & =\left(\sum_{\substack{i, j=1 \\
i \neq j}}^{n} \frac{w_{i} w_{j}}{1-w_{i}} a_{i}^{p} a_{j}^{q}\right)^{1 /(p+q)} \\
& =\left(\frac{1}{n(n-1)} \sum_{\substack{i, j=1 \\
i \neq j}}^{n} a_{i}^{p} a_{j}^{q}\right)^{1 /(p+q)}=\operatorname{BM}^{p, q}\left(a_{1}, a_{2}, \ldots, a_{n}\right),
\end{aligned}
$$

which complete the proof of the property.

Property 2 (Idempotency). Let $p, q \geq 0$ and $a_{i}(i=1,2, \ldots, n)$ be a collection of nonnegative numbers with the weight vector $w=\left(w_{1}, w_{2}, \ldots, w_{n}\right)$, such that $w_{i} \geq 0$, and $\sum_{i=1}^{n} w_{i}=1$. If all $a_{i}(i=1,2, \ldots, n)$ are equal, that is, $a_{i}=a$, for all $i$, then

$$
\operatorname{NWBM}^{p, q}\left(a_{1}, a_{2}, \ldots, a_{n}\right)=a
$$


Proof. Since $a_{i}=a(i=1,2, \ldots, n)$, then

$$
\begin{aligned}
\operatorname{NWBM}^{p, q}\left(a_{1}, a_{2}, \ldots, a_{n}\right) & =\left(\sum_{i=1}^{n} w_{i} a^{p} \sum_{\substack{j=1 \\
j \neq i}}^{n} \frac{w_{j}}{1-w_{i}} a^{q}\right)^{1 /(p+q)} \\
& =\left(a^{p+q} \sum_{i=1}^{n} w_{i} \sum_{\substack{j=1 \\
j \neq i}}^{n} \frac{w_{j}}{1-w_{i}}\right)^{1 /(p+q)}=a,
\end{aligned}
$$

which complete the proof of the property.

Property 3 (Monotonicity). Let $p, q \geq 0, a_{i}$ and $b_{i}(i=1,2, \ldots, n)$ be two collections of nonnegative numbers with the weight vector $w=\left(w_{1}, w_{2}, \ldots, w_{n}\right)$, such that $w_{i} \geq 0$ and $\sum_{i=1}^{n} w_{i}=1$. If $a_{i} \geq b_{i}$, for all $i$, then

$$
\operatorname{NWBM}^{p, q}\left(a_{1}, a_{2}, \ldots, a_{n}\right) \geq \operatorname{NWBM}^{p, q}\left(b_{1}, b_{2}, \ldots, b_{n}\right) .
$$

Proof. Since $a_{i} \geq b_{i}$ for all $i$, and $p, q \geq 0$, then

$$
\begin{gathered}
a_{i}^{p} a_{j}^{q} \geq b_{i}^{p} b_{j}^{q} \quad(i, j=1,2, \ldots, n), \\
\left(\sum_{\substack{i, j=1 \\
i \neq j}}^{n} \frac{w_{i} w_{j}}{1-w_{i}} a_{i}^{p} a_{j}^{q}\right) \geq\left(\sum_{\substack{i, j=1 \\
i \neq j}}^{n} \frac{w_{i} w_{j}}{1-w_{i}} b_{i}^{p} b_{j}^{q}\right) .
\end{gathered}
$$

Therefore,

$$
\operatorname{NWBM}^{p, q}\left(a_{1}, a_{2}, \ldots, a_{n}\right) \geq \operatorname{NWBM}^{p, q}\left(b_{1}, b_{2}, \ldots, b_{n}\right)
$$

which complete the proof.

Property 4 (Boundedness). Let $p, q \geq 0$ and $a_{i}(i=1,2, \ldots, n)$ be a collection of nonnegative numbers with the weight vector $w=\left(w_{1}, w_{2}, \ldots, w_{n}\right)$, such that $w_{i} \geq 0$, and $\sum_{i=1}^{n} w_{i}=1$, then

$$
\min _{i}\left\{a_{i}\right\} \leq \operatorname{NWBM}^{p, q}\left(a_{1}, a_{2}, \ldots, a_{n}\right) \leq \max _{i}\left\{a_{i}\right\}
$$

Proof. By Property 2, we can get

$$
\begin{aligned}
& \operatorname{NWBM}^{p, q}\left(\min _{i}\left\{a_{i}\right\}, \min _{i}\left\{a_{i}\right\}, \ldots, \min _{i}\left\{a_{i}\right\}\right)=\min _{i}\left\{a_{i}\right\}, \\
& \operatorname{NWBM}^{p, q}\left(\max _{i}\left\{a_{i}\right\}, \max _{i}\left\{a_{i}\right\}, \ldots, \max _{i}\left\{a_{i}\right\}\right)=\max _{i}\left\{a_{i}\right\} .
\end{aligned}
$$


Since $\min _{i}\left\{a_{i}\right\} \leq a_{i} \leq \max _{i}\left\{a_{i}\right\}(i=1,2, \ldots, n)$, then based on Property 3 , we have

$$
\min _{i}\left\{a_{i}\right\} \leq \operatorname{NWBM}^{p, q}\left(a_{1}, a_{2}, \ldots, a_{n}\right) \leq \max _{i}\left\{a_{i}\right\}
$$

which complete the proof of the theorem.

\subsection{GNWMB}

In this subsection, we further extend the NWBM to the generalized normalized weighted Bonferroni mean (GNWBM) by considering the correlation of any three aggregated arguments instead of any two based on the GBM

Definition 3.2. Let $p, q, r \geq 0$, and $a_{i}(i=1,2, \ldots, n)$ be a collection of nonnegative numbers with the weight vector $w=\left(w_{1}, w_{2}, \ldots, w_{n}\right)$ such that $w_{i} \geq 0$, and $\sum_{i=1}^{n} w_{i}=1$. If

$$
\operatorname{GNWBM}^{p, q, r}\left(a_{1}, a_{2}, \ldots, a_{n}\right)=\left(\sum_{\substack{i, j, k=1 \\ i \neq j \neq k}}^{n} \frac{w_{i} w_{j} w_{k}}{\left(1-w_{i}\right)\left(1-w_{i}-w_{j}\right)} a_{i}^{p} a_{j}^{q} a_{k}^{r}\right)^{1 /(p+q+r)},
$$

then $\mathrm{GNWBM}^{p, q, r}$ is called the generalized normalized weighted Bonferroni mean (GNWBM).

Furthermore, we can transform the GNWBM into the interrelationship GNWBM form as follows:

$$
\operatorname{GNWBM}^{p, q, r}\left(a_{1}, a_{2}, \ldots, a_{n}\right)=\left(\sum_{i=1}^{n} w_{i} a_{i}^{p} \sum_{\substack{j=1 \\ j \neq i}}^{n} \frac{w_{j}}{1-w_{i}} a_{j}^{q} \sum_{\substack{k=1 \\ k \neq i \neq j}}^{n} \frac{w_{k}}{\left(1-w_{i}-w_{j}\right)} a_{k}^{r}\right)^{1 /(p+q+r)}
$$

We see that the term $\sum_{j=1, j \neq i}^{n}\left(w_{j} /\left(1-w_{i}\right)\right) a_{j}^{q}$ is the weighted power average satisfaction of all criteria except $A_{i}$, with $\sum_{j=1, j \neq i}^{n}\left(w_{j} /\left(1-w_{i}\right)\right)=1$. The term $\sum_{k=1, k \neq i \neq j}^{n}\left(w_{k} /(1-\right.$ $\left.\left.w_{i}-w_{j}\right)\right) a_{k}^{r}$ is the weighted power average satisfaction of all criteria except $A_{i}$ and $A_{j}$, with $\sum_{k=1, k \neq i \neq j}^{n}\left(w_{k} /\left(1-w_{i}-w_{j}\right)\right)=1$. Here then $\mathrm{NWBM}^{p, q}$ represents the interrelationship between any three aggregated arguments, which is similar to the GBM. Especially, if $r \rightarrow 0$, then the GNWBM reduces to the NWBM.

Moreover, the GNWBM has the following properties.

Property 5 (Reducibility). Let $p, q, r \geq 0$ and $a_{i}(i=1,2, \ldots, n)$ be a collection of nonnegative numbers with the weight vector $w=(1 / n, 1 / n, \ldots 1 / n)$, then

$$
\operatorname{GNWBM}^{p, q, r}\left(a_{1}, a_{2}, \ldots, a_{n}\right)=\operatorname{GBM}^{p, q, r}\left(a_{1}, a_{2}, \ldots, a_{n}\right)
$$


Proof. Since $w=(1 / n, 1 / n, \ldots 1 / n)$, then by Definition 3.2, we can get

$$
\begin{aligned}
\operatorname{GNWBM}^{p, q, r}\left(a_{1}, a_{2}, \ldots, a_{n}\right) & =\left(\sum_{\substack{i, j, k=1 \\
i \neq j \neq k}}^{n} \frac{1}{n(n-1)(n-2)} a_{i}^{p} a_{j}^{q} a_{k}^{r}\right)^{1 /(p+q+r)} \\
& =\left(\frac{1}{n(n-1)(n-2)} \sum_{\substack{i, j, k=1 \\
i \neq j \neq k}}^{n} a_{i}^{p} a_{j}^{q} a_{k}^{r}\right)^{1 /(p+q+r)}, \\
\operatorname{GNWBM}^{p, q, r}\left(a_{1}, a_{2}, \ldots, a_{n}\right)=\operatorname{GBM}^{p, q, r}\left(a_{1}, a_{2}, \ldots, a_{n}\right), &
\end{aligned}
$$

which complete the proof of the property.

Property 6 (Idempotency). Let $p, q, r \geq 0$ and $a_{i}(i=1,2, \ldots, n)$ be a collection of nonnegative numbers with the weight vector $w=\left(w_{1}, w_{2}, \ldots, w_{n}\right)$, such that $w_{i} \geq 0$ and $\sum_{i=1}^{n} w_{i}=1$. If all $a_{i}(i=1,2, \ldots, n)$ are equal, that is, $a_{i}=a$, for all $i$, then

$$
\operatorname{GNWBM}^{p, q, r}\left(a_{1}, a_{2}, \ldots, a_{n}\right)=a \text {. }
$$

Proof. The proof of Property 6 is similar to Property 2.

Property 7 (Monotonicity). Let $p, q, r \geq 0 a_{i}$ and $b_{i}(i=1,2, \ldots, n)$ be two collections of nonnegative numbers with the weight vector $w=\left(w_{1}, w_{2}, \ldots, w_{n}\right)$, such that $w_{i} \geq 0$ and $\sum_{i=1}^{n} w_{i}=1$. If $a_{i} \geq b_{i}$, for all $i$, then

$$
\operatorname{GNWBM}^{p, q, r}\left(a_{1}, a_{2}, \ldots, a_{n}\right) \geq \operatorname{GNWBM}^{p, q, r}\left(b_{1}, b_{2}, \ldots, b_{n}\right) .
$$

Proof. The proof of Property 7 is similar to Property 3.

Property 8 (Boundedness). Let $p, q, r \geq 0$ and $a_{i}(i=1,2, \ldots, n)$ be a collection of nonnegative numbers with the weight vector $w=\left(w_{1}, w_{2}, \ldots, w_{n}\right)$, such that $w_{i} \geq 0$ and $\sum_{i=1}^{n} w_{i}=1$, then

$$
\min _{i}\left\{a_{i}\right\} \leq \operatorname{GNWBM}^{p, q, r}\left(a_{1}, a_{2}, \ldots, a_{n}\right) \leq \max _{i}\left\{a_{i}\right\} .
$$

Proof. The proof of Property 8 is similar to Property 4.

\section{Intuitionistic Fuzzy Normalized Weighted BM and Generalized Intuitionistic Fuzzy Normalized Weighted BM}

To aggregate the intuition fuzzy correlated information, $\mathrm{Xu}$ and Yager [19] proposed the IFBM and IFWBM, and Xia et al. [21] proposed the GIFWBM. However, according to the aforementioned analysis, there are some drawbacks in the IFWBM and the GIFWBM, respectively. To solve these issues, and motivated by the GBM, we propose the intuitionistic 
fuzzy normalized weighted BM (IFNWBM) and the generalized intuitionistic fuzzy normalized weighted BM (GIFNWBM) based on the NWBM and GNWBM and describe as follows.

\subsection{IFNWBM}

Definition 4.1. Let $p, q \geq 0$ and $\alpha_{i}=\left(\mu_{i}, v_{i}\right)(i=1,2, \ldots, n)$ be a collection of IFVs with the weight vector $w=\left(w_{1}, w_{2}, \ldots, w_{n}\right)$ such that $w_{i} \geq 0$ and $\sum_{i=1}^{n} w_{i}=1$. If

$$
\operatorname{IFNWBM}^{p, q}\left(\alpha_{1}, \alpha_{2}, \ldots, \alpha_{n}\right)=\left(\bigoplus_{i, j=1, i \neq j}^{n} \frac{w_{i} w_{j}}{1-w_{i}}\left(\alpha_{i}^{p} \otimes \alpha_{j}^{q}\right)\right)^{1 /(p+q)}
$$

then IFNWBM ${ }^{p, q}$ is called the intuitionistic fuzzy normalized weighted Bonferroni mean (IFNWBM).

On the basis of the operational laws of IFVs, we have the following theorem.

Theorem 4.2. Let $p, q \geq 0$ and $\alpha_{i}=\left(\mu_{i}, v_{i}\right)(i=1,2, \ldots, n)$ be a collection of IFVs with the weight vector $\left(w_{1}, w_{2}, \ldots, w_{n}\right)$, such that $w_{i} \geq 0$ and $\sum_{i=1}^{n} w_{i}=1$, then the aggregated value by using the IFNWBM is also an IFV and

$$
\begin{aligned}
& \operatorname{IFNWBM}^{p, q}\left(\alpha_{1}, \alpha_{2}, \ldots, \alpha_{n}\right) \\
&=\left(\left(1-\prod_{\substack{i, j=1 \\
i \neq j}}^{n}\left(1-\mu_{i}^{p} \mu_{j}^{q}\right)^{w_{i} w_{j} /\left(1-w_{i}\right)}\right)^{1 /(p+q)},\right. \\
&\left.1-\left(1-\prod_{\substack{i, j=1 \\
i \neq j}}^{n}\left(1-\left(1-v_{i}\right)^{p}\left(1-v_{j}\right)^{q}\right)^{w_{i} w_{j} /\left(1-w_{i}\right)}\right)^{1 /(p+q)}\right) .
\end{aligned}
$$

Proof. By the operational laws for IFVs, we have

$$
\begin{gathered}
\alpha_{i}^{p}=\left(\mu_{i}^{p}, 1-\left(1-v_{i}\right)^{p}\right), \quad \alpha_{j}^{q}=\left(\mu_{j}^{q}, 1-\left(1-v_{j}\right)^{q}\right), \\
\alpha_{i}^{p} \otimes \alpha_{j}^{q}=\left(\mu_{i}^{p} \mu_{j}^{q}, 1-\left(1-v_{i}\right)^{p}\left(1-v_{j}\right)^{q}\right),
\end{gathered}
$$


then

$$
\begin{aligned}
\bigoplus_{i, j=1, i \neq j}^{n} \frac{w_{i} w_{j}}{1-w_{i}}\left(\alpha_{i}^{p} \otimes \alpha_{j}^{q}\right)= & \left(1-\prod_{\substack{i, j=1 \\
i \neq j}}^{n}\left(1-\mu_{i}^{p} \mu_{j}^{q}\right)^{w_{i} w_{j} /\left(1-w_{i}\right)}, \prod_{\substack{i, j=1 \\
i \neq j}}^{n}\left(1-\left(1-v_{i}\right)^{p}\left(1-v_{j}\right)^{q}\right)^{w_{i} w_{j} /\left(1-w_{i}\right)}\right) \\
\operatorname{IFNWBM}^{p, q}\left(\alpha_{1}, \alpha_{2}, \ldots, \alpha_{n}\right)= & \left(\bigoplus_{i, j=1, i \neq j}^{n} \frac{w_{i} w_{j}}{1-w_{i}}\left(\alpha_{i}^{p} \otimes \alpha_{j}^{q}\right)\right)^{1 /(p+q)}, \\
= & \left(\left(1-\prod_{\substack{i, j=1 \\
i \neq j}}^{n}\left(1-\mu_{i}^{p} \mu_{j}^{q}\right)^{w_{i} w_{j} /\left(1-w_{i}\right)}\right)^{1 /(p+q)},\right. \\
& \left(1-\left(\begin{array}{c}
\left.1-\prod_{\substack{i, j=1 \\
i \neq j}}^{n}\left(1-\left(1-v_{i}\right)^{p}\left(1-v_{j}\right)^{q}\right)^{w_{i} w_{j} /\left(1-w_{i}\right)}\right)^{1 /(p+q)}
\end{array}\right) .\right.
\end{aligned}
$$

In addition, since

$$
\begin{array}{r}
0 \leq\left(1-\prod_{\substack{i, j=1 \\
i \neq j}}^{n}\left(1-\mu_{i}^{p} \mu_{j}^{q}\right)^{w_{i} w_{j} /\left(1-w_{i}\right)}\right)^{1 /(p+q)} \leq 1, \\
0 \leq 1-\left(1-\prod_{\substack{i, j=1 \\
i \neq j}}^{n}\left(1-\left(1-v_{i}\right)^{p}\left(1-v_{j}\right)^{q}\right)^{w_{i} w_{j} /\left(1-w_{i}\right)}\right)^{1 /(p+q)} \leq 1,
\end{array}
$$

then

$$
\begin{aligned}
& \left(1-\prod_{\substack{i, j=1 \\
i \neq j}}^{n}\left(1-\mu_{i}^{p} \mu_{j}^{q}\right)^{w_{i} w_{j} /\left(1-w_{i}\right)}\right)^{1 /(p+q)} \\
& +1-\left(1-\prod_{\substack{i, j=1 \\
i \neq j}}^{n}\left(1-\left(1-v_{i}\right)^{p}\left(1-v_{j}\right)^{q}\right)^{w_{i} w_{j} /\left(1-w_{i}\right)}\right)^{1 /(p+q)}
\end{aligned}
$$


Journal of Applied Mathematics

$$
\begin{aligned}
\leq & 1+\left(1-\prod_{\substack{i, j=1 \\
i \neq j}}^{n}\left(1-\left(1-v_{i}\right)^{p}\left(1-v_{j}\right)^{q}\right)^{w_{i} w_{j} /\left(1-w_{i}\right)}\right)^{1 /(p+q)} \\
& -\left(1-\prod_{\substack{i, j=1 \\
i \neq j}}^{n}\left(1-\left(1-v_{i}\right)^{p}\left(1-v_{j}\right)^{q}\right)^{w_{i} w_{j} /\left(1-w_{i}\right)}\right)^{1 /(p+q)}=1,
\end{aligned}
$$

which completes the proof of the theorem.

Moreover, the IFNWBM also has the following properties.

Property 9. If all $\alpha_{\mathrm{i}}(i=1,2, \ldots, n)$ are equal, that is, $\alpha_{i}=\alpha$, for all $i$, then

$$
\operatorname{IFNWBM}^{p, q}\left(\alpha_{1}, \alpha_{2}, \ldots, \alpha_{n}\right)=\alpha
$$

Proof. Since $\alpha_{i}=\left(\mu_{i}, v_{i}\right)=\alpha$, we have

$$
\begin{aligned}
& \operatorname{IFNWBM}^{p, q}\left(\alpha_{1}, \alpha_{2}, \ldots, \alpha_{n}\right) \\
& =\left(\bigoplus_{i, j=1, i \neq j}^{n} \frac{w_{i} w_{j}}{1-w_{i}}\left(\alpha^{p} \otimes \alpha^{q}\right)\right)^{1 /(p+q)}=\left(\left(\alpha^{p} \otimes \alpha^{q}\right) \sum_{\substack{i, j=1 \\
i \neq j}}^{n} \frac{w_{i} w_{j}}{1-w_{i}}\right)^{1 /(p+q)} \\
& =\alpha\left(\sum_{i}^{n} w_{i} \sum_{\substack{j=1 \\
j \neq i}}^{n} \frac{w_{j}}{1-w_{i}}\right)^{1 /(p+q)}=\alpha,
\end{aligned}
$$

which completes the proof.

Property 10. Let $\alpha_{i}=\left(\mu_{\alpha_{i}}, v_{\alpha_{i}}\right)$ and $\beta_{i}=\left(\mu_{\beta_{i}}, v_{\beta_{i}}\right)(i=1,2, \ldots, n)$ be two collections of IFVs, if $\mu_{\alpha_{i}} \geq \mu_{\beta_{i}}$ and $v_{\alpha_{i}} \leq v_{\beta_{i}}$, for all $i$, then

$$
\operatorname{IFNWBM}^{p, q}\left(\alpha_{1}, \alpha_{2}, \ldots, \alpha_{n}\right) \geq \operatorname{IFNWBM}^{p, q}\left(\beta_{1}, \beta_{2}, \ldots, \beta_{n}\right)
$$


Proof. Since $\mu_{\alpha_{i}} \geq \mu_{\beta_{i}}$ and $v_{\alpha_{i}} \leq v_{\beta_{i}}$, for all $i$, then

$$
\begin{aligned}
& \mu_{\alpha_{i}}^{p} \mu_{\alpha_{j}}^{q} \geq \mu_{\beta_{i}}^{p} \mu_{\beta_{j}}^{q} \\
& \Longrightarrow \prod_{\substack{i, j=1 \\
i \neq j}}^{n}\left(1-\mu_{\alpha_{i}}^{p} \mu_{\alpha_{j}}^{q}\right)^{w_{i} w_{j} /\left(1-w_{i}\right)} \leq \prod_{\substack{i, j=1 \\
i \neq j}}^{n}\left(1-\mu_{\beta_{i}}^{p} \mu_{\beta_{j}}^{q}\right)^{w_{i} w_{j} /\left(1-w_{i}\right)} \\
& \Longrightarrow 1-\prod_{\substack{i, j=1 \\
i \neq j}}^{n}\left(1-\mu_{\alpha_{i}}^{p} \mu_{\alpha_{j}}^{q}\right)^{w_{i} w_{j} /\left(1-w_{i}\right)} \geq 1-\prod_{\substack{i, j=1 \\
i \neq j}}^{n}\left(1-\mu_{\beta_{i}}^{p} \mu_{\beta_{j}}^{q}\right)^{w_{i} w_{j} /\left(1-w_{i}\right)} \\
& \Longrightarrow\left(1-\prod_{\substack{i, j=1 \\
i \neq j}}^{n}\left(1-\mu_{\alpha_{i}}^{p} \mu_{\alpha_{j}}^{q}\right)^{w_{i} w_{j} /\left(1-w_{i}\right)}\right)^{1 /(p+q)} \\
& \geq\left(1-\prod_{\substack{i, j=1 \\
i \neq j}}^{n}\left(1-\mu_{\beta_{i}}^{p} \mu_{\beta_{j}}^{q}\right)^{w_{i} w_{j} /\left(1-w_{i}\right)}\right)^{1 /(p+q)}, \\
& \left(1-v_{\alpha_{i}}^{p}\right)\left(1-v_{\alpha_{j}}^{q}\right) \geq\left(1-v_{\beta_{i}}^{p}\right)\left(1-v_{\beta_{j}}^{q}\right) \\
& \Longrightarrow \prod_{\substack{i, j=1 \\
i \neq j}}^{n}\left(1-\left(1-v_{\alpha_{i}}^{p}\right)\left(1-v_{\alpha_{j}}^{q}\right)\right)^{w_{i} w_{j} /\left(1-w_{i}\right)} \\
& \leq \prod_{\substack{i, j=1 \\
i \neq j}}^{n}\left(1-\left(1-v_{\beta_{i}}^{p}\right)\left(1-v_{\beta_{j}}^{q}\right)\right)^{w_{i} w_{j} /\left(1-w_{i}\right)} \\
& \Longrightarrow\left(1-\prod_{\substack{i, j=1 \\
i \neq j}}^{n}\left(1-\left(1-v_{\alpha_{i}}^{p}\right)\left(1-v_{\alpha_{j}}^{q}\right)\right)^{w_{i} w_{j} /\left(1-w_{i}\right)}\right)^{1 /(p+q)} \\
& \geq\left(1-\prod_{\substack{i, j=1 \\
i \neq j}}^{n}\left(1-\left(1-v_{\beta_{i}}^{p}\right)\left(1-v_{\beta_{j}}^{q}\right)\right)^{w_{i} w_{j} /\left(1-w_{i}\right)}\right)^{1 /(p+q)},
\end{aligned}
$$

then

$$
\begin{aligned}
& 1-\left(1-\prod_{\substack{i, j=1 \\
i \neq j}}^{n}\left(1-\left(1-v_{\alpha_{i}}^{p}\right)\left(1-v_{\alpha_{j}}^{q}\right)\right)^{w_{i} w_{j} /\left(1-w_{i}\right)}\right)^{1 /(p+q)} \\
& \quad \leq 1-\left(1-\prod_{\substack{i, j=1 \\
i \neq j}}^{n}\left(1-\left(1-v_{\beta_{i}}^{p}\right)\left(1-v_{\beta_{j}}^{q}\right)\right)^{w_{i} w_{j} /\left(1-w_{i}\right)}\right)^{1 /(p+q)} .
\end{aligned}
$$


Therefore,

$$
\begin{aligned}
& \left(1-\prod_{\substack{i, j=1 \\
i \neq j}}^{n}\left(1-\mu_{\alpha_{i}}^{p} \mu_{\alpha_{j}}^{q}\right)^{w_{i} w_{j} /\left(1-w_{i}\right)}\right)^{1 /(p+q)}-1+\left(1-\prod_{\substack{i, j=1 \\
i \neq j}}^{n}\left(1-\left(1-v_{\alpha_{i}}^{p}\right)\left(1-v_{\alpha_{j}}^{q}\right)\right)^{w_{i} w_{j} /\left(1-w_{i}\right)}\right)^{1 /(p+q)} \\
& \quad \geq\left(1-\prod_{\substack{i, j=1 \\
i \neq j}}^{n}\left(1-\mu_{\beta_{i}}^{p} \mu_{\beta_{j}}^{q}\right)^{w_{i} w_{j} /\left(1-w_{i}\right)}\right)^{1 /(p+q)}-1 \\
& \\
& \quad+\left(1-\prod_{\substack{i, j=1 \\
i \neq j}}^{n}\left(1-\left(1-v_{\beta_{i}}^{p}\right)\left(1-v_{\beta_{j}}^{q}\right)\right)^{w_{i} w_{j} /\left(1-w_{i}\right)}\right)^{1 /(p+q)} .
\end{aligned}
$$

Let $\alpha=\operatorname{IFNWBM}^{p, q}\left(\alpha_{1}, \alpha_{2}, \ldots, \alpha_{n}\right)$ and $\beta=\operatorname{IFNWBM}^{p, q}\left(\beta_{1}, \beta_{2}, \ldots, \beta_{n}\right)$ and set $s(\alpha)$ and $s(\beta)$ be the score values of $\alpha$ and $\beta$, then (4.12) is equal to $s(\alpha) \geq s(\beta)$. Now we discuss the following two cases.

Case 1. If $s(\alpha)>s(\beta)$, then by the total order relation between two IFVs, we have

$$
\operatorname{IFNWBM}^{p, q}\left(\alpha_{1}, \alpha_{2}, \ldots, \alpha_{n}\right)>\operatorname{IFNWBM}^{p, q}\left(\beta_{1}, \beta_{2}, \ldots, \beta_{n}\right)
$$

Case 2. If $s(\alpha)=s(\beta)$, then

$$
\begin{aligned}
(1- & \left.\prod_{\substack{i, j=1 \\
i \neq j}}^{n}\left(1-\mu_{\alpha_{i}}^{p} \mu_{\alpha_{j}}^{q}\right)^{w_{i} w_{j} /\left(1-w_{i}\right)}\right)^{1 /(p+q)}-1+\left(1-\prod_{\substack{i, j=1 \\
i \neq j}}^{n}\left(1-\left(1-v_{\alpha_{i}}^{p}\right)\left(1-v_{\alpha_{j}}^{q}\right)\right)^{w_{i} w_{j} /\left(1-w_{i}\right)}\right)^{1 /(p+q)} \\
& =\left(1-\prod_{\substack{i, j=1 \\
i \neq j}}^{n}\left(1-\mu_{\beta_{i}}^{p} \mu_{\beta_{j}}^{q}\right)^{w_{i} w_{j} /\left(1-w_{i}\right)}\right)^{1 /(p+q)}-1 \\
& +\left(1-\prod_{\substack{i, j=1 \\
i \neq j}}^{n}\left(1-\left(1-v_{\beta_{i}}^{p}\right)\left(1-v_{\beta_{j}}^{q}\right)\right)^{w_{i} w_{j} /\left(1-w_{i}\right)}\right)^{1 /(p+q)} .
\end{aligned}
$$


Since $\mu_{\alpha_{i}} \geq \mu_{\beta_{i}}$ and $v_{\alpha_{i}} \leq v_{\beta_{i}}$, for all $i$, we can get

$$
\begin{gathered}
\left(1-\prod_{\substack{i, j=1 \\
i \neq j}}^{n}\left(1-\mu_{\alpha_{i}}^{p} \mu_{\alpha_{j}}^{q}\right)^{w_{i} w_{j} /\left(1-w_{i}\right)}\right)^{1 /(p+q)}=\left(1-\prod_{\substack{i, j=1 \\
i \neq j}}^{n}\left(1-\mu_{\beta_{i}}^{p} \mu_{\beta_{j}}^{q}\right)^{w_{i} w_{j} /\left(1-w_{i}\right)}\right)^{1 /(p+q)}, \\
1-\left(1-\prod_{\substack{i, j=1 \\
i \neq j}}^{n}\left(1-\left(1-v_{\alpha_{i}}^{p}\right)\left(1-v_{\alpha_{j}}^{q}\right)\right)^{w_{i} w_{j} /\left(1-w_{i}\right)}\right)^{1 /(p+q)} \\
=1-\left(1-\prod_{\substack{i, j=1 \\
i \neq j}}^{n}\left(1-\left(1-v_{\beta_{i}}^{p}\right)\left(1-v_{\beta_{j}}^{q}\right)\right)^{w_{i} w_{j} /\left(1-w_{i}\right)}\right)^{1 /(p+q)} .
\end{gathered}
$$

Therefore, $h(\alpha)=h(\beta)$ and

$$
\operatorname{IFNWBM}^{p, q}\left(\alpha_{1}, \alpha_{2}, \ldots, \alpha_{n}\right)=\operatorname{IFNWBM}^{p, q}\left(\beta_{1}, \beta_{2}, \ldots, \beta_{n}\right)
$$

which complete the proof of the property.

Property 11. Let $\alpha_{i}=\left(\mu_{i}, v_{j}\right)(i=1,2, \ldots, n)$ be a collection of IFVs, and $\left(\widetilde{\alpha}_{1}, \widetilde{\alpha}_{2}, \ldots, \widetilde{\alpha}_{n}\right)$ is any permutation of $\left(\alpha_{1}, \alpha_{2}, \ldots, \alpha_{n}\right)$, then

$$
\operatorname{IFNWBM}^{p, q}\left(\tilde{\alpha}_{1}, \tilde{\alpha}_{2}, \ldots, \tilde{\alpha}_{n}\right)=\operatorname{IFNWBM}^{p, q}\left(\alpha_{1}, \alpha_{2}, \ldots, \alpha_{n}\right) \text {. }
$$

Proof. Since $\left(\tilde{\alpha}_{1}, \tilde{\alpha}_{2}, \ldots, \widetilde{\alpha}_{n}\right)$ is any permutation of $\left(\alpha_{1}, \alpha_{2}, \ldots, \alpha_{n}\right)$, then

$$
\left(\bigoplus_{\substack{i, j=1 \\ i \neq j}}^{n} \frac{w_{i} w_{j}}{1-w_{i}}\left(\alpha_{i}^{p} \otimes \alpha_{j}^{q}\right)\right)^{1 /(p+q)}=\left(\bigoplus_{\substack{i, j=1 \\ i \neq j}}^{n} \frac{w_{i} w_{j}}{1-w_{i}}\left(\tilde{\alpha}_{i}^{p} \otimes \widetilde{\alpha}_{j}^{q}\right)\right)^{1 /(p+q)} .
$$

Therefore,

$$
\operatorname{IFNWBM}^{p, q}\left(\alpha_{1}, \alpha_{2}, \ldots, \alpha_{n}\right)=\operatorname{IFNWBM}^{p, q}\left(\widetilde{\alpha}_{1}, \widetilde{\alpha}_{2}, \ldots, \widetilde{\alpha}_{n}\right),
$$

which complete the proof. 
Property 12. Let $\alpha_{i}=\left(\mu_{i}, v_{j}\right)(i=1,2, \ldots, n)$ be a collection of IFVs, and

$$
\alpha^{-}=\left(\min _{i}\left\{\mu_{i}\right\}, \max _{i}\left\{v_{i}\right\}\right), \quad \alpha^{+}=\left(\max _{i}\left\{\mu_{i}\right\}, \min _{i}\left\{v_{i}\right\}\right),
$$

then

$$
\alpha^{-} \leq \operatorname{IFNWBM}^{p, q}\left(\alpha_{1}, \alpha_{2}, \ldots, \alpha_{n}\right) \leq \alpha^{+} .
$$

Proof. Since $\mu_{i} \geq \min _{i}\left\{\mu_{i}\right\}$ and $v_{i} \leq \max _{i}=\left\{v_{i}\right\}$, then based on Properties 9 and 10, we have

$$
\operatorname{IFNWBM}^{p, q}\left(\alpha_{1}, \alpha_{2}, \ldots, \alpha_{n}\right) \geq \operatorname{IFNWBM}^{p, q}\left(\alpha^{-}, \alpha^{-}, \ldots, \alpha^{-}\right)=\alpha^{-}
$$

Likewise, we can get

$$
\operatorname{IFNWBM}^{p, q}\left(\alpha_{1}, \alpha_{2}, \ldots, \alpha_{n}\right) \leq \operatorname{IFNWBM}^{p, q}\left(\alpha^{+}, \alpha^{+}, \ldots, \alpha^{+}\right)=\alpha^{+},
$$

which complete the proof of the property.

\subsection{GIFNWBM}

Definition 4.3. Let $p, q, r \geq 0$ and $\alpha_{i}=\left(\mu_{i}, v_{i}\right)(i=1,2, \ldots, n)$ be a collection of IFVs with the weight vector $w=\left(w_{1}, w_{2}, \ldots, w_{n}\right)$ such that $w_{i} \geq 0$, and $\sum_{i=1}^{n} w_{i}=1$. If

$$
\operatorname{GIFNWBM}^{p, q, r}\left(\alpha_{1}, \ldots, \alpha_{n}\right)=\left(\bigoplus_{\substack{i, j, k=1 \\ i \neq j \neq k}}^{n} \frac{w_{i} w_{j} w_{k}}{\left(1-w_{i}\right)\left(1-w_{i}-w_{j}\right)}\left(\alpha_{i}^{p} \otimes \alpha_{j}^{q} \otimes \alpha_{k}^{r}\right)\right)^{1 /(p+q+r)}
$$

then GIFNWBM ${ }^{p, q, r}$ is called the generalized intuitionistic fuzzy normalized weighted Bonferroni mean (GIFNWBM). 
On the basis of the operational laws of IFVs, we can drive the following theorem.

Theorem 4.4. Let $p, q, r \geq 0$ and $\alpha_{i}=\left(\mu_{i}, v_{i}\right)(i=1,2, \ldots, n)$ be a collection of IFVs with the weight vector $w=\left(w_{1}, w_{2}, \ldots, w_{n}\right)$, such that $w_{i} \geq 0$ and $\sum_{i=1}^{n} w_{i}=1$, then the aggregated value by using the GIFNWBM is also an IFV and

$$
\begin{aligned}
\operatorname{IFNWBM} & \\
= & \left(\left(1-\prod_{\substack{i, j, k=1 \\
i \neq j \neq k}}^{n}\left(1-\mu_{i}^{p} \mu_{j}^{q} \mu_{k}^{r}\right)^{w_{i} w_{j} w_{k} /\left(\left(1-w_{i}\right)\left(1-w_{i}-w_{j}\right)\right)}\right)^{1 /(p+q+r)},\right. \\
& \left.1-\left(1-\prod_{\substack{i, j, k=1 \\
i \neq j \neq k}}^{n}\left(1-\left(1-v_{i}\right)^{p}\left(1-v_{j}\right)^{q}\left(1-v_{k}\right)^{r}\right)^{w_{i} w_{j} w_{k} /\left(\left(1-w_{i}\right)\left(1-w_{i}-w_{j}\right)\right)}\right)^{1 /(p+q+r)}\right)
\end{aligned}
$$

Proof. The proof of Theorem 4.4 is similar to Theorem 4.2.

Furthermore, the GIFNWBM also has the following properties.

Property 13. If all $\alpha_{i}(i=1,2, \ldots, n)$ are equal, that is, $\alpha_{i}=\alpha$, for all $i$, then

$$
\operatorname{GIFNWBM}^{p, q, r}\left(\alpha_{1}, \alpha_{2}, \ldots, \alpha_{n}\right)=\alpha
$$

Proof. The proof of Property 13 is similar to Property 9.

Property 14. Let $\alpha_{i}=\left(\mu_{\alpha_{i}}, v_{\alpha_{i}}\right)$ and $\beta_{i}=\left(\mu_{\beta_{i}}, v_{\beta_{i}}\right)(i=1,2, \ldots, n)$ be two collections of IFVs, if $\mu_{\alpha_{i}} \geq \mu_{\beta_{i}}$ and $v_{\alpha_{i}} \leq v_{\beta_{i}}$, for all $i$, then

$$
\operatorname{GIFNWBM}^{p, q, r}\left(\alpha_{1}, \alpha_{2}, \ldots, \alpha_{n}\right) \geq \operatorname{GIFNWBM}^{p, q, r}\left(\beta_{1}, \beta_{2}, \ldots, \beta_{n}\right)
$$

Proof. The proof of Property 14 is similar to Property 10.

Property 15. Let $\alpha_{i}=\left(\mu_{i}, v_{j}\right)(i=1,2, \ldots, n)$ be a collection of IFVs and $\left(\tilde{\alpha}_{1}, \tilde{\alpha}_{2}, \ldots, \tilde{\alpha}_{n}\right)$ is any permutation of $\left(\alpha_{1}, \alpha_{2}, \ldots, \alpha_{n}\right)$, then

$$
\operatorname{GIFNWBM}^{p, q, r}\left(\widetilde{\alpha}_{1}, \tilde{\alpha}_{2}, \ldots, \tilde{\alpha}_{n}\right)=\operatorname{GIFNWBM}^{p, q, r}\left(\alpha_{1}, \alpha_{2}, \ldots, \alpha_{n}\right)
$$

Proof. The proof of Property 15 is similar to Property 11.

Property 16. Let $\alpha_{i}=\left(\mu_{i}, v_{j}\right)(i=1,2, \ldots, n)$ be a collection of IFVs, and

$$
\alpha^{-}=\left(\min _{i}\left\{\mu_{i}\right\}, \max _{i}\left\{v_{i}\right\}\right), \quad \alpha^{+}=\left(\max _{i}\left\{\mu_{i}\right\}, \min _{i}\left\{v_{i}\right\}\right),
$$


then

$$
\alpha^{-} \leq \operatorname{GIFNWBM}^{p, q, r}\left(\alpha_{1}, \alpha_{2}, \ldots, \alpha_{n}\right) \leq \alpha^{+} .
$$

Proof. The proof of Property 16 is similar to Property 12.

\section{An Approach to Intuitionistic Fuzzy Multicriteria Decision Making}

In what follows, we apply the GIFNWBM operator to intuitionistic fuzzy multicriteria decision making, which involves the following steps.

Step 1. For a multicriteria decision making problem, set $Y=\left\{y_{1}, y_{2}, \ldots, y_{n}\right\}$ be a set of $n$ alternatives, $C=\left\{c_{1}, c_{2}, \ldots, c_{m}\right\}$ be a set of $m$ criteria, whose weight vector is $w=$ $\left(w_{1}, w_{2}, \ldots, w_{m}\right)$, satisfying $w_{j}>0(j=1,2, \ldots, m)$, and $\sum_{j=1}^{m} w_{j}=1$, where $w_{j}$ denotes the important degree of $c_{j}$. The performance of $y_{i}$ with respect to $c_{j}$ is measured by an IFV $b_{i j}=\left(\mu_{i j}, v_{i j}\right)$, where $\mu_{i j}$ indicates the degree that $y_{i}$ satisfies $c_{j}$ and $v_{i j}$ indicates the degree that $y_{i}$ does not satisfy $c_{j}$, such that $0 \leq \mu_{i j} \leq 1,0 \leq v_{i j} \leq 1$ and $\mu_{i j}+v_{i j} \leq 1$, and the intuitionistic fuzzy decision matrix $B=\left(b_{i j}\right)_{n \times m}$ contains all $b_{i j}=\left(\mu_{i j}, v_{i j}\right)(i=1,2, \ldots, n ; j=1,2, \ldots, m)$. If all the criteria $c_{j}$ are the benefit type, then the performance values do not need normalization. Whereas there are, generally, benefit criteria (the bigger the performance values the better) and cost criteria (the smaller the performance values the better) in multicriteria decision making, in such cases, we may transform the performance values of the cost type into the performance values of the benefit type. by $\mathrm{Xu}$ and $\mathrm{Hu}$ 's approach [26]. Then, the intuitionistic fuzzy decision matrix $B=\left(b_{i j}\right)_{n \times m}$ can be transformed into the normalization matrix $R=$ $\left(r_{i j}\right)_{n \times m}$ where

$$
\left(r_{i j}\right)_{n \times m}=\left(\mu_{i j}^{\prime}, v_{i j}^{\prime}\right)= \begin{cases}b_{i j}, & \text { for benefit criterion } c_{i}, \\ \bar{b}_{i j}, & \text { for cost criterion } c_{i},\end{cases}
$$

where $i=1,2, \ldots, n, j=1,2, \ldots, m$, and $\bar{b}_{i j}$ is the complement of $b_{i j}$ such that $\bar{b}_{i j}=\left(v_{i j}, \mu_{i j}\right)$.

Step 2. Utilize the GIFNWBM operator:

$$
r_{i}=\left(\mu_{i}^{\prime}, v_{i}^{\prime}\right)=\operatorname{GIFNWBM}^{p, q, r}\left(r_{i 1}, r_{i 2}, \ldots, r_{i m}\right) .
$$

to aggregate all the preference values $r_{i j}(j=1,2, \ldots, m)$ of the $i$ th line and get the overall performance value $r_{i}$ corresponding to the alternative $y_{i}$.

Step 3. Calculate the score valued and the accuracy degree of the overall performance value $r_{i}$ and utilize the total order relation between two IFVs to rank the overall performance value $r_{i}(i=1,2, \ldots, n)$.

Step 4. Rank all the alternatives $y_{i}(i=1,2, \ldots, n)$ in accordance with $r_{i}(i=1,2, \ldots, n)$ in descending order, and then, select the most desirable alternative with the largest score value. 
Table 1: Normalization intuitionistic fuzzy decision matrix $R=\left(r_{i j}\right)_{4 \times 5}$.

\begin{tabular}{cccccc}
\hline & $c_{1}$ & $c_{2}$ & $c_{3}$ & $c_{4}$ & $c_{5}$ \\
\hline$y_{1}$ & $(0.85,0.12)$ & $(0.90,0.08)$ & $(0.80,0.07)$ & $(0.55,0.30)$ & $(0.83,0.15)$ \\
$y_{2}$ & $(0.69,0.28)$ & $(0.78,0.19)$ & $(0.75,0.20)$ & $(0.85,0.10)$ & $(0.65,0.30)$ \\
$y_{3}$ & $(0.72,0.24)$ & $(0.85,0.12)$ & $(0.82,0.16)$ & $(0.40,0.35)$ & $(0.95,0.03)$ \\
$y_{4}$ & $(0.63,0.35)$ & $(0.60,0.25)$ & $(0.65,0.30)$ & $(0.94,0.02)$ & $(0.90,0.00)$ \\
\hline
\end{tabular}

Let us give a practical example to illustrate the proposed approach in the intuitionistic fuzzy multicriteria decision making procedure.

Example 5.1. There is an investment company, which wants to invest a sum of money in the best option (adapted from Herrera and Herrera-Viedma [27]). There is a panel with four possible alternatives $\left\{y_{1}, y_{2}, y_{3}, y_{4}\right\}$ to invest the money, in which $y_{1}$ is a car company, $y_{2}$ is a fast food chains company, $y_{3}$ is an arms company, $y_{4}$ is a software company. The investment company must make a decision according to five criteria: $c_{1}$ is the growth analysis, $c_{2}$ is the environment impact analysis, $c_{3}$ is the risk analysis, $c_{4}$ is the social impact analysis, $c_{5}$ is the profitability analysis. The weight vector of the criteria $\left\{c_{1}, c_{2}, c_{3}, c_{4}, c_{5}\right\}$ is $w=$ $(0.15,0.12,0.24,0.18,0.31)$. Assume that the characteristics of the alternatives $y_{i}(i=1,2,3,4)$ with respect to the criteria $c_{j}(j=1,2,3,4,5)$ are represented by the IFVs $b_{i j}=\left(\mu_{i j}, v_{i j}\right)$, where $\mu_{i j}$ indicates the degree that the alternative $y_{i}$ satisfies the criterion $c_{j}$ and $v_{i j}$ indicates the degree that the alternative $y_{i}$ does not satisfy the criterion $c_{j}$.

To get the optimal alternative(s), the following steps are given.

Step 1. Based on (5.1), we normalize $b_{i j}(i=1,2,3,4 ; j=1,2,3,4,5)$ to $r_{i j}$ and construct the normalization intuitionistic fuzzy decision matrix $R=\left(r_{i j}\right)_{4 \times 5}$ (see Table 1 ).

Step 2. Aggregate all the preference values $r_{i j}(j=1,2,3,4,5)$ of the $i$ th line, and get the overall performance value $r_{i}$ corresponding to the alternative $y_{i}$ by the GIFNWBM operator (here we let $p=q=r=1$ ):

$$
\begin{array}{ll}
r_{1}=(0.6331,0.2910), & r_{2}=(0.5891,0.3692), \\
r_{3}=(0.6056,0.3233), & r_{4}=(0.6049,0.3222) .
\end{array}
$$

Step 3. Calculate the score of the overall performance value $r_{i}(i=1,2,3,4)$ :

$$
s\left(r_{1}\right)=0.3421, \quad s\left(r_{2}\right)=0.2199, \quad s\left(r_{3}\right)=0.2823, \quad s\left(r_{4}\right)=0.2827
$$

Step 4. Rank all the alternatives $y_{i}(i=1,2,3,4)$ in accordance with $r_{i}$. Since $s\left(r_{1}\right)>s\left(r_{4}\right)>$ $s\left(r_{3}\right)>s\left(r_{2}\right)$, then by the total order relation between two IFVs, we have the ranking of the IFVs: $y_{1}>y_{4}>y_{3}>y_{2}$. Hence, $y_{1}$ is the best option.

In Step 2, if we take $p=q=r=2$, we can get

$$
\begin{array}{ll}
r_{1}=(0.6974,0.2335), & r_{2}=(0.6510,0.3079), \\
r_{3}=(0.6770,0.2627), & r_{4}=(0.6697,0.2604) .
\end{array}
$$


Then, we calculate the score values of all the alternatives:

$$
s\left(r_{1}\right)=0.4640, \quad s\left(r_{2}\right)=0.3430, \quad s\left(r_{3}\right)=0.4143, \quad s\left(r_{4}\right)=0.4093 .
$$

Therefore, $y_{1}>y_{3}>y_{4}>y_{2}$, and $y_{1}$ is still the optimal alternative.

By the aforementioned numeral results, the optimal investment decision is the car company $y_{1}$. It should be noted out that the whole ranking of the alternatives has changed. The GIFNWBM ${ }^{1,1,1}$ produces the ranking of all the alternatives as $y_{1}>y_{4}>y_{3}>y_{2}$, which is slightly different from the ranking of alternatives $y_{1}>y_{3}>y_{4}>y_{2}$, derived by the GIFNWBM ${ }^{2,2,2}$, that is, the ranking of $y_{3}$ and $y_{4}$ is reversed while the ranking of the other alternatives is kept unchanged. Therefore, we can see that the value derived by the GIFNWBM operator depends on the choice of the parameters $p, q$, and $r$, and these parameters are not robust. In general, the bigger parameters $p, q$, and $r$, the more the calculation effort needed, and in the special case where at least two of these parameters take the value of zero, the GIFNWBM cannot capture the interrelationship of the individual arguments. As a result, in practical applications, we generally take the values of these parameters as $p=q=r=1$, which is not only intuitive and simple but also the interrelationship of the individual argument can be fully taken into account [21].

\section{Concluding Remarks}

To aggregate the intuitionistic fuzzy information, a lot of aggregation operators have been developed and investigated, especially, the ones which reflect the interrelationship of the aggregated arguments are the hot research topics, among which the Bonferroni mean (BM) is an important aggregation technique. The desirable characteristic of the BM is its capability to capture the interrelationship between the input arguments. To further develop the $\mathrm{BM}$, we have proposed the normalized weighted Bonferroni mean (NWBM) and the generalized normalized weighted Bonferroni mean (GNWBM) whose characteristics are to reflect the preference and interrelationship of the aggregated arguments and can satisfy the basic properties of the aggregation techniques simultaneously. To aggregate the IFVs, the intuitionistic fuzzy normalized weighted Bonferroni mean (IFNWBM) operator and the generalized intuitionistic fuzzy normalized weighted Bonferroni mean (GIFNWBM) operator have been developed and discussed. Furthermore, some desirable properties of the IFNWBM operator and the GIFNWBM operator are investigated in detail. To deal with the situation that the criteria have connections in intuitionistic fuzzy multicriteria decision making, an approach has been proposed on the basis of the GIFNWBM operator. It is worth noting that the results of this paper can be extended to the interval-valued intuitionistic fuzzy environment and the hesitant fuzzy environment.

\section{Acknowledgments}

This work was supported by National Natural Science Foundation of China (no. 71071034), National Basic Research Program of China (973 Program, no. 2010 CB328104-02), Funding of Jiangsu Innovation Program for Graduate Education (no. CXZZ-0183), and Academic New Artist Ministry of Education Doctoral Post Graduate. 


\section{References}

[1] G. Beliakov, S. James, J. Mordelová, T. Rückschlossová, and R. R. Yager, “Generalized Bonferroni mean operators in multi-criteria aggregation," Fuzzy Sets and Systems, vol. 161, no. 17, pp. 2227-2242, 2010.

[2] V. Torra and Y. Narukawa, Modeling Decisions: Information Fusion and Aggregation Operators, Springer, Berlin, Germany, 2007.

[3] R. Mesiar, A. Kolesarova, T. Calvo, and M. Komornikova, "A review of aggregation functions," in Fuzzy Sets and Their Extensions: Representation, Aggregation and Models, H. Bustince, F. Herrera, and J. Montero, Eds., Springer, Heidelberg, Germany, 2008.

[4] M. Grabisch, J.-L. Marichal, R. Mesiar, and E. Pap, Aggregation Functions, Cambridge University Press, Cambridge, UK, 2009.

[5] C. Bonferroni, "Sulle medie multiple di potenze," Bollettino Della Unione Matematica Italiana, vol. 5, no. 3-4, pp. 267-270, 1950.

[6] R. R. Yager, “On generalized Bonferroni mean operators for multi-criteria aggregation," International Journal of Approximate Reasoning, vol. 50, no. 8, pp. 1279-1286, 2009.

[7] J. J. Dujmović and H. L. Larsen, "Generalized conjunction/disjunction," International Journal of Approximate Reasoning, vol. 46, no. 3, pp. 423-446, 2007.

[8] J. J. Dujmović, "Continuous preference logic for system evaluation," IEEE Transactions on Fuzzy Systems, vol. 15, no. 6, pp. 1082-1099, 2007.

[9] J.-L. Marichal, "k-intolerant capacities and Choquet integrals," European Journal of Operational Research, vol. 177, no. 3, pp. 1453-1468, 2007.

[10] G. Choquet, "Theory of capacities," Annales de l'Institut Fourier, vol. 5, pp. 131-295, 1955.

[11] R. R. Yager, “On ordered weighted averaging aggregation operators in multicriteria decision making," IEEE Transactions on Systems, Man, and Cybernetics, vol. 18, no. 1, pp. 183-190, 1988.

[12] J. Mordelová and T. Rückschlossová, "ABC-aggregation functions," in Proceedings of the 5th International Summer School on Aggregation Operators, pp. 167-170, Palmade Mallorca, Spain, 2009.

[13] L. A. Zadeh, "Fuzzy sets," Information and Control, vol. 8, no. 3, pp. 338-353, 1965.

[14] L. A. Zadeh, "The concept of a linguistic variable and its application to approximate reasoning," Information Sciences, vol. 8, pp. 199-249, 1975.

[15] K. T. Atanassov, "Intuitionistic fuzzy sets," Fuzzy Sets and Systems, vol. 20, no. 1, pp. 87-96, 1986.

[16] K. T. Atanassov and G. Gargov, "Interval valued intuitionistic fuzzy sets," Fuzzy Sets and Systems, vol. 31, no. 3, pp. 343-349, 1989.

[17] V. Torra and Y. Narukawa, "On hesitant fuzzy sets and decision," in Proceedings of the 18th IEEE International Conference on Fuzzy Systems, pp. 1378-1382, Jeju Island, Korea, August 2009.

[18] V. Torra, "Hesitant fuzzy sets," International Journal of Intelligent Systems, vol. 25, no. 6, pp. 529-539, 2010.

[19] Z. S. Xu and R. R. Yager, "Intuitionistic fuzzy bonferroni means," IEEE Transactions on Systems, Man, and Cybernetics, vol. 41, no. 2, pp. 568-578, 2011.

[20] Z. S. Xu and Q. Chen, "A multi-criteria decision making procedure based on interval-valued intuitionistic fuzzy bonferroni means," Journal of Systems Science and Systems Engineering, vol. 20, no. 2, pp. 217-228, 2011.

[21] M. M. Xia, Z. S. Xu, and B. Zhu, "Generalized intuitionistic fuzzy Bonferroni means," International Journal of Intelligent Systems, vol. 27, no. 1, pp. 23-47, 2012.

[22] Z. S. Xu, "Intuitionistic fuzzy aggregation operators," IEEE Transactions on Fuzzy Systems, vol. 15, no. 6, pp. 1179-1187, 2007.

[23] S. M. Chen and J. M. Tan, "Handling multicriteria fuzzy decision-making problems based on vague set theory," Fuzzy Sets and Systems, vol. 67, no. 2, pp. 163-172, 1994.

[24] D. H. Hong and C. H. Choi, "Multicriteria fuzzy decision-making problems based on vague set theory," Fuzzy Sets and Systems, vol. 114, no. 1, pp. 103-113, 2000.

[25] Z. S. Xu and R. R. Yager, "Some geometric aggregation operators based on intuitionistic fuzzy sets," International Journal of General Systems, vol. 35, no. 4, pp. 417-433, 2006.

[26] Z. S. Xu and H. Hu, "Projection models for intuitionistic fuzzy multiple attribute decision making," International Journal of Information Technology and Decision Making, vol. 9, no. 2, pp. 267-280, 2010.

[27] F. Herrera and E. Herrera-Viedma, "Linguistic decision analysis: steps for solving decision problems under linguistic information," Fuzzy Sets and Systems, vol. 115, no. 1, pp. 67-82, 2000. 


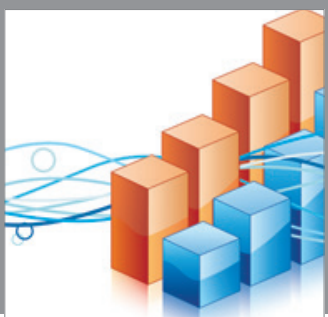

Advances in

Operations Research

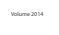

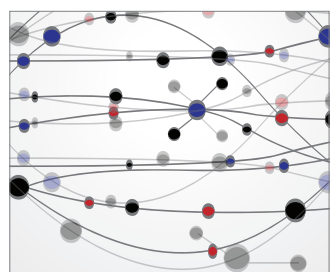

\section{The Scientific} World Journal
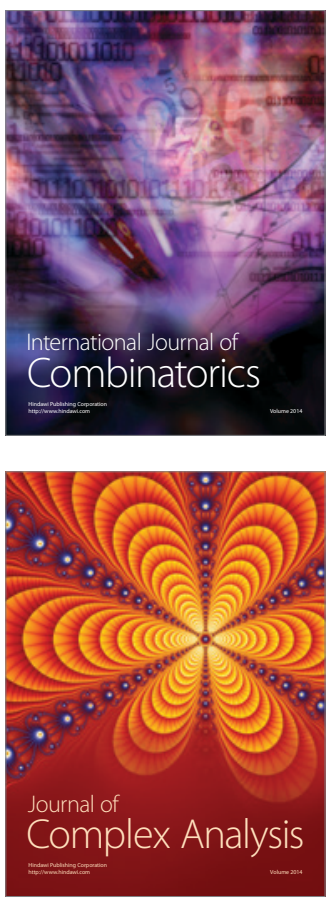

International Journal of

Mathematics and

Mathematical

Sciences
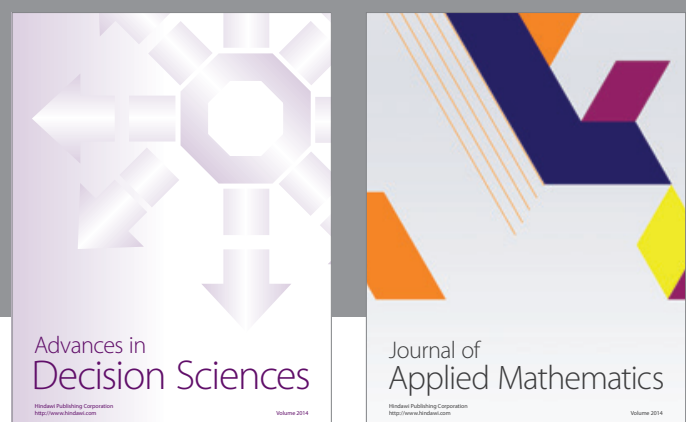

Journal of

Applied Mathematics
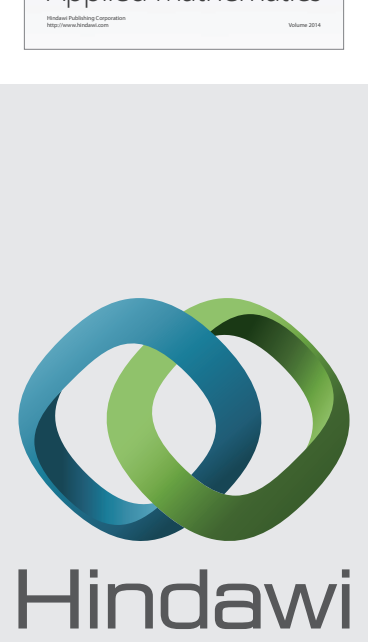

Submit your manuscripts at http://www.hindawi.com
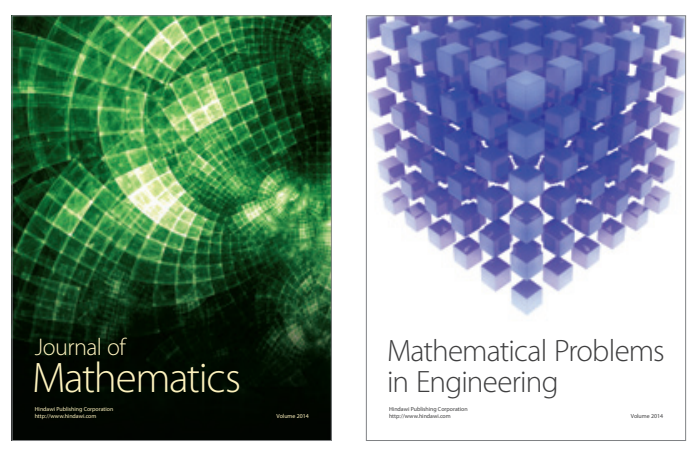

Mathematical Problems in Engineering
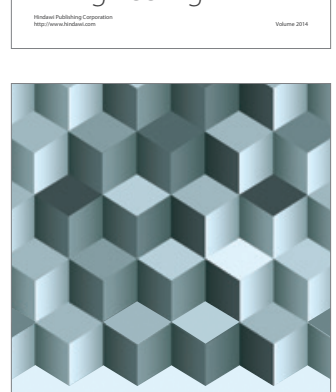

Journal of

Function Spaces
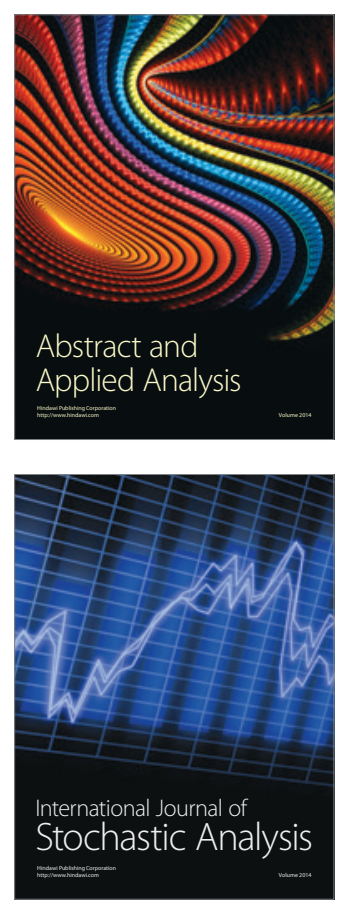

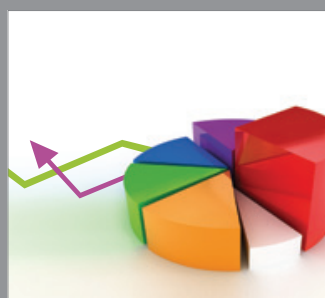

ournal of

Probability and Statistics

Promensencen
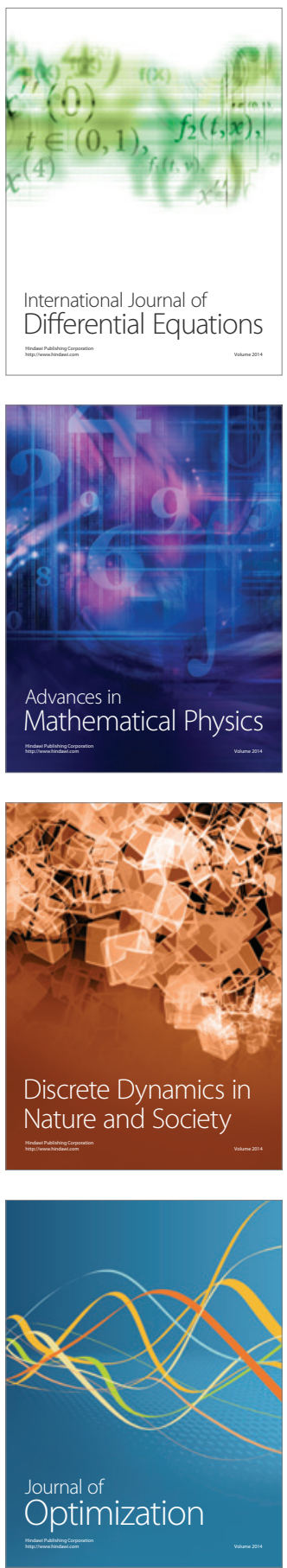